\title{
Origin and Evolution of the Cepheus Bubble
}

\author{
N.A. Patel ${ }^{1}$, P.F. Goldsmith ${ }^{2}$, M.H. Heyer ${ }^{3}$, R. Snell ${ }^{3}$, and P. Pratap ${ }^{3}$ \\ 1 SAO, Cambridge, MA, USA \\ 2 NAIC, Cornell University, Ithaca, NY, USA \\ ${ }^{3}$ FCRAO, University of Massachusetts, Amherst, MA, USA
}

\begin{abstract}
The Cepheus bubble is a giant shell with a diameter of about $120 \mathrm{pc}$, discovered by Kun (1987) from IRAS sky flux maps. We have imaged this $10^{\circ} \times 10^{\circ}$ region in the $\mathrm{CO} J=1-0$ line, using the FCRAO Quarry system with sample spacing equal to the 45" FWHM beam width. We estimate the total molecular gas mass to be $\sim 10^{5}$ solar masses and a total kinetic energy $\sim 10^{51}$ ergs. We find $49 \mathrm{O}$ and $\mathrm{B} 0-$ B2 type stars in the Cepheus bubble region, most belong to the CepOB2 association. 120 IRAS point sources, selected according to color criteria representative of young stellar objects, fall within our map. Many of these sources are associated with the globules and filamentary structures seen in the $\mathrm{CO}$ emission, similar to the situation found in IC1396 (Patel et al. 1995). These stars, which are still associated with dense molecular gas plausibly represent the third and youngest generation of stars in this region.
\end{abstract}

\section{Introduction}

The Cepheus bubble is similar in size to the Local Bubble. Its relative proximity (distance $\sim 800 \mathrm{pc}$, Ábráham et al. 1993) makes it a good object for a molecular line study in order to understand the structure and kinematics of the gas associated with the bubble and the phenomenon of sequentially triggered star formation. Here we briefly present some of the results from our CO mapping of this region, with the hope of providing an example of a bubble that can be compared in physical characteristics and phenomena with those of the Local Bubble.

\section{Observations \& Results}

The observations consist of CO 1-0 emission line mapping carried out using the Five College Radio Astronomy Observatory $14 \mathrm{~m}$ telescope at New Salem, Massachusetts, with the QUARRY focal plane array receiver. The $1 \mathrm{MHz}$ per channel filterbank provided a velocity coverage of about $100 \mathrm{~km} / \mathrm{s}$ centered upon $-10 \mathrm{~km} / \mathrm{s} \mathrm{V}_{\mathrm{LSR}}$. Observations were made in position switching mode. Integration time was varying between 20 to 30 seconds per pointing, resulting in an rms noise of about 0.1 to $0.2 \mathrm{~K}$. The total area covered by the map is about 72 square degrees, sampled every 50 " with a FWHM resolution of 45 ", resulting in about 387,000 spectra using a total telescope time of about 260 
hours. The mapping boundaries were based upon the IRAS emission maps. Figure 1. shows the peak intensity map with antenna temperature in the velocity range $-28-+9 \mathrm{~km} / \mathrm{s} V_{\mathrm{LSR}}$. The features in Figure 1 which appear like filaments or globules, show values of antenna temperature typically between 2 and $8 \mathrm{~K}$, whereas the diffuse clouds appear significantly fainter. In the latter, we are likely to be under-resolving the CO lines with our $3 \mathrm{~km} / \mathrm{s}$ velocity resolution.

We have selected $\mathrm{O}$ and up to B2 type stars from the Position and Proper Motions Catalog (Roeser \& Bastian 1988) and found 49 stars, including 6 O type stars belonging to the CepOB2 association. The $\mathrm{O}$ stars themselves appear to form a smaller ring within the larger $\mathrm{CO}$ and infrared ring. We have also selected IRAS points sources according to their IRAS color criteria as suggested by Beichman, Boulanger and Moshir (1992) to represent young stellar objects, as in our previous work on IC1396 (Patel et al. 1995). We found 120 IRAS point sources in the field of the Cepheus bubble satisfying these color criteria. 50 of them are directly coincident upon the globules and filamentary clouds. The diffuse clouds are noticeably lacking in IRAS sources.

From the $21 \mathrm{~cm}$ line observations of neutral atomic hydrogen, made at the Dominion Radio Observatory's $26 \mathrm{~m}$ telescope, we estimate a total atomic gas mass to be of the same order as the molecular gas: about $3 \times 10^{5} \mathrm{M}_{\odot}$. Within the uncertainties involved in the determination of both $\mathrm{CO}$ and HI masses, we can conclude that they are of comparable masses. Thus, the total gas mass associated with the Cepheus bubble is about $4 \times 10^{5} \mathrm{M}_{\odot}$. The total kinetic energy in the system of molecular clouds associated with the Cepheus bubble is about $10^{51} \mathrm{ergs}$ (Patel et al. 1997).

\section{Interpretation}

Since the center of the bubble is well above the galactic plane, the expansion of the shell due to supernova explosion must proceed in an asymmetrical way. Numerical calculations taking into account of this effect, have been presented by Silich (1996). (See also Bisnovatyi-Kogan, these proceedings).

The Cepheus bubble region has been influenced by energy input from a previous generation of massive stars as suggested by the following observations. (1) As noted by Kun et al. (1987), the existence of the run-away star, $\lambda$ Cep suggests that its binary companion could have exploded as a supernova somewhere in the central region of the Cepheus bubble. Another run-away star, $68 \mathrm{Cyg}$ is also likely to be arising from Cep OB 2 region (Blaauw 1961). (2) The O stars (and many B stars) appear to form a ring on their own, in Figure 1, which is smaller than the Cepheus bubble. (3) Around Cep OB2, there appears a significant population of evolved massive stars such as $\mu$ Ceph (M2), $\nu$ Ceph (A2), VV Ceph (M2), belong to an older association with an age of about $10^{7}$ years (Simonson and van Someren Green 1976) (4) The properties of dust in and around IC1396 suggest that it has been pre-heated 
due to shocks presumably due to supernovae (Clayton \& Fitzpatrick 1987). (5) As noted in our work on IC1396 (Patel et al. 1995), the region outside of the ring of globules also appears to be very effectively evacuated of neutral gas; which is consistent with an event preceding the onset of the formation of the exciting star of IC1396.

The massive stars of this previous generation, would also have led to an expanding shell of gas in the parent molecular cloud in which they were born. This shell, initially expanding adiabatically, would gradually become gravitationally unstable, and lead to the condensations resulting in formation of the second generation of stars (Comerón \& Torra 1994; McCray \& Kafatos 1987).

We estimate the time scale for this instability as well as the size, velocity of expansion and the collected mass of the shell at which the instability occurs, by solving numerically the equation of motion of the shell following Mazurek (1980) and Maddalena \& Morris (1987). We obtain $10 \mathrm{~cm}^{-3}$ for the molecular gas density and $8.0 \times 10^{47} \mathrm{~s}^{-1}$ for the luminosity of the driving source, for such a shell to become unstable on reaching a radius of about $30 \mathrm{pc}$ which is roughly the radius of the ring defined by the $O$ type stars in Figure 1. The instability occurs at an age of about 7 Myrs and the mass accumulated by such a shell is about $6.0 \times 10^{4} \mathrm{M}_{\odot}$.

\section{Conclusions}

The observational characteristics of the Cepheus bubble are consistent with stellar wind and supernovae energy input due to an earlier generation of $O B$ stars, over a period of about $10^{7} \mathrm{yrs}$, which roughly agrees with the age of NGC 7160 (Leisawitz et al. 1989). CepOB2 may have formed as a consequence of gravitational instability in the expanding shell around this 1st generation of OB stars. The molecular cloud kinematics show evidence for expansion away from the galactic plane. A third, and youngest, generation of low to intermediate mass stars appear to be forming in the globules and filamentary clouds of the Cepheus bubble.

\section{References}

Ábráham, P., Kun, M., Balázs, Holl, A. \& Fronto, A., 1993, A\&A 268, 230

Beichman, C. A., Boulanger, F. \& Moshir, M., (1992), ApJ 386, 248

Blaauw, A., 1961, Bull. Astron. Inst. Neth., 15, 265

Clayton, G.C. \& Fitzpatrick, E.L., 1987, AJ 92, 1

Comerón, F. \& Torra, J., 1994, ApJ 423, 652

Kun, M., Balazs, L. G. \& Toth, I., 1987, Astrophys. Sp. Sci. 134, 211

Leisawitz, D., Bash, F.N. \& Thaddeus, P., 1989, ApJSS 70, 731

Maddelena, R.J. \& Morris, M., 1987, ApJ 323, 179

Mazurek T.J., 1980, A\&A 90, 65 


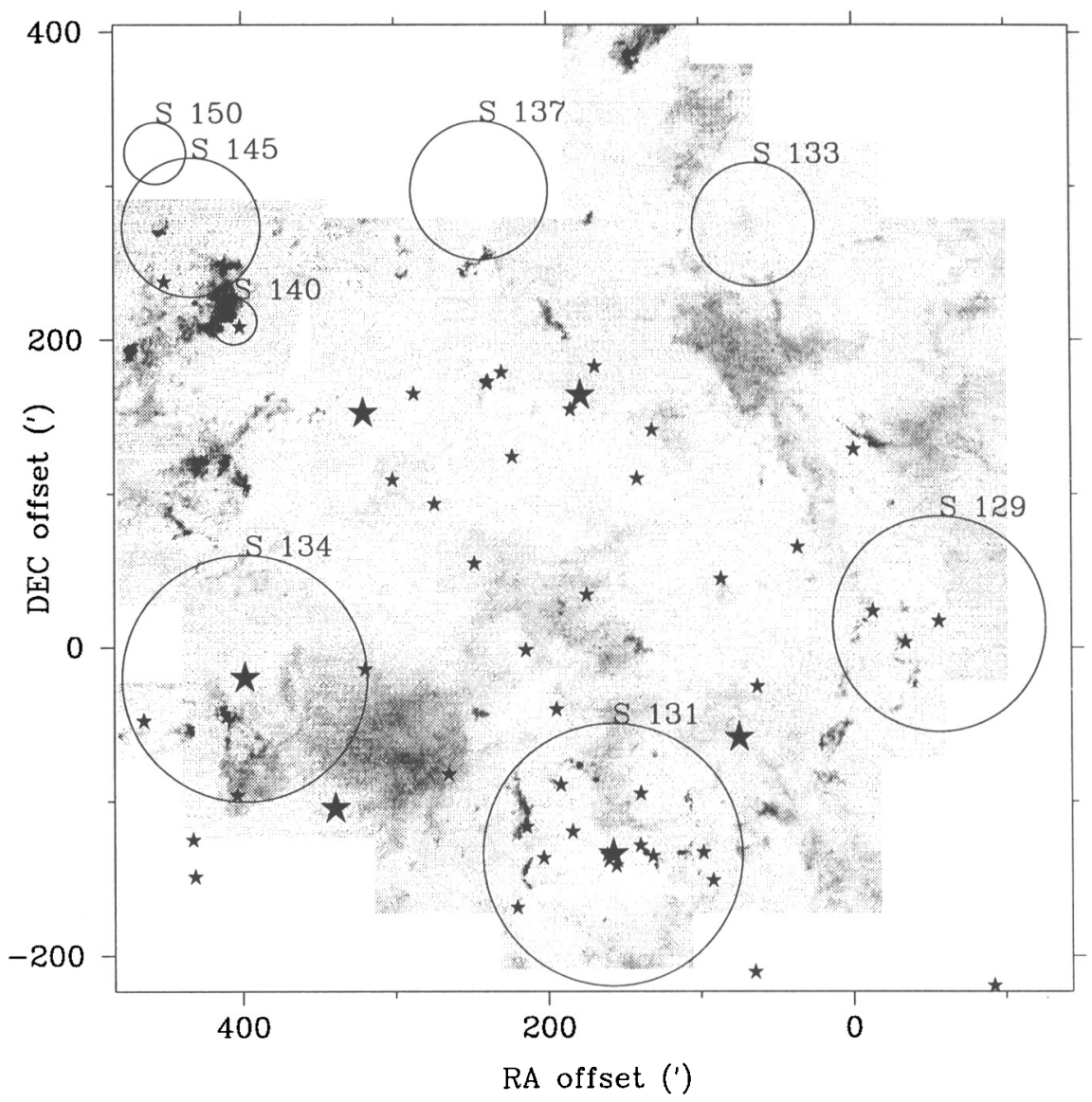

Fig. 1. Peak intensity of CO 1-0 emission. The grey scale represents antenna temperature values scaled linearly between 0 and $3.5 \mathrm{~K}$. The strongest emission occurs at the S140 region and globule $A$ of IC1396, where the line peak antenna temperature is about $10 \mathrm{~K}$. A map of the integrated emission appears similar to this figure and is therefore excluded. The position offsets are measured from $\alpha(1950)=21^{h} 18^{m} 00^{s}, \delta(1950)=59^{\circ} 30^{\prime} 00^{\prime \prime}$, near S129.

McCray, R., \& Kafatos, M., 1987, ApJ 317, 190

Patel N.A., Goldsmith, P.F., Snell, R.L., Hezel T., Xie, T., 1995, ApJ 447, 721

Patel N.A., Goldsmith, P.F., Heyer, M.H., Snell, R.L., Pratap, P., 1997, in preparation.

Roeser, S. \& Bastian, U., 1988, A\&ASS 74, 449

Silich, S. .A., 1996, ApJ 468, 722

Simonson, S.C.III, \& van Someren Greve, H.W., 1976, A\&A 49, 343 